\title{
Shear model with shear-flexure interaction for non-linear analysis of reinforced concrete frame element
}

\author{
Worathep Sae-Long ${ }^{1, *}$, and Suchart Limkatanyu ${ }^{1}$ \\ ${ }^{1}$ Department of Civil Engineering, Faculty of Engineering, Prince of Songkla University, Hat Yai, Songkhla, Thailand
}

\begin{abstract}
This paper presents the shear constitutive model for the reinforced concrete $(\mathrm{R} / \mathrm{C})$ frame structures analysis under monotonic and cyclic loading. The proposed model is adopted and modified from Mergos and Koppos model [1] that accounts the shear stiffness degradation effect by the shear-flexure interaction in the plastic hinge region. Firstly, the proposed shear model starts from the primary curve without the damages due to the shear-flexure interaction effect. Then, the shear-flexure interaction effect is taken into consideration at the locations of plastic hinges and this effect leads to the degradation of the shear strength and shear stiffness on the undamaged primary curve that is replaced with the damaged primary curve. To determine the sectional shear stiffness with the shear-flexure interaction, an alternative way of the iterative procedure is proposed here. Finally, a numerical example is used to verify the characteristics and behavior of the $\mathrm{R} / \mathrm{C}$ frame system and confirm accuracy and computational efficiency of the proposed model among the experimental data.
\end{abstract}

\section{Introduction}

The reinforced concrete structures (R/C-Structure) have been used extensively in engineering works, for example, the building and bridge structures etc. From the past earthquakes, the $\mathrm{R} / \mathrm{C}$ members before the proposed seismic codes were often collapsed and heavily damaged due to the member without ductile design requirement. The most dangerous damage mechanism was related to shear that occurred suddenly because of the insufficient shear strength. However, the characteristics of $\mathrm{R} / \mathrm{C}$ member failure didn't account only the shear failure but also included the flexural failure and the flexure-shear failure [2].

The flexure-shear failure might be significant in some cases such as the lightly $\mathrm{R} / \mathrm{C}$ columns. To represent the accurate responses of those members, there were several researchers [2-3] who developed the analytical procedures to include the shear-flexure interaction into the assessment of element by using the different strategies. Although these procedures were attractive, these methods were not successful with the degradation of shear strength in the plastic hinge region. Later, Mergos and Kappos [1,4] presented the shear model and the shear-flexure interaction procedure that was able to capture the degradation of the shear strength in the plastic hinges region. However, although these methods were attractive, it didn't seem reasonable in the real analysis because their method approximated the shear stiffness with the shear-flexure interaction which based on a constant stiffness in every step.

In the light of this reason, this paper proposes an alternative of a shear model with the modified technique for the degraded shear stiffness estimation due to the shear-flexure interaction effect. Moreover, a numerical example is used to confirm the accuracy and efficiency of the proposed model. The example presents the member with failing in shear after the flexural yielding that is defined to the shear-flexure critical member. All the responses of the model in this paper are implemented in the general-purpose finite element platform FEAP [5].

\section{Shear model}

The shear model represents the shear force-shear strain relation of the $\mathrm{R} / \mathrm{C}$ member. The original shear model was proposed by Mergos and Kappos [1] and their model covered all the behaviors of $\mathrm{R} / \mathrm{C}$ member prior and after concrete cracking, the flexural yielding and the transverse reinforcement yielding or failure as indicated in the primary curve for this paper.

\subsection{Primary curve of shear model}

The primary curve presents the initial shear force-shear strain relation that is first derived without considering the damage due to the shear-flexure interaction effect. As shown in Fig. 1, it comprises four branches with three different slopes. The first branch oa with uncracked slope $\left(G A_{0}\right)$ shows the elastic behavior of uncracked section in shear. The origin point o connects to the cracking point a $\left(V_{c r}, \gamma_{c r}\right)$ at which the nominal principal tensile stress becomes larger than the nominal tensile strength of concrete. The cracking shear force $\left(V_{c r}\right)$ [4], 
the uncracked slope $\left(G A_{0}\right)$ and the cracking shear strain $\left(\gamma_{c r}\right)$ can be written as:

$$
\begin{gathered}
V_{c r}=\frac{f_{t}^{\prime}}{L_{a} / h} \sqrt{1+\frac{N}{f_{t}^{\prime} A_{g}}} 0.80 A_{g} \\
G A_{0}=0.80 G A_{g} \\
\gamma_{c r}=V_{c r} / G A_{0}
\end{gathered}
$$

Where $f_{t}^{\prime}$ is the mean concrete tensile strength, $A_{g}$ is the gross area of the concrete section, $L_{a}$ is the shear span, $h$ is the section height, $N$ is the compressive axial load and $G$ is the shear modulus respectively.

The second branch ab and third branch bc have the same slope of $G A_{1}$. The second branch ab links the cracking point a $\left(V_{c r}, \gamma_{c r}\right)$ to the flexural-yielding point $\mathrm{b}$ $\left(V_{y}, \gamma_{y}\right)$ at which the longitudinal reinforcement yields for the first time. The third branch bc joins the flexuralyielding point $\mathrm{b}\left(V_{y}, \gamma_{y}\right)$ to the shear-yielding point $\mathrm{c}$ $\left(V_{u 0}, \gamma_{s t}\right)$ at which shear strain corresponds to the onset of the transverse reinforcement yielding $\left(\gamma_{s t}\right)$ while shear force reaches its ultimate value $\left(V_{u 0}\right)$.

To estimate the shear strength $\left(V_{u}\right)$ on the primary curve, the shear strength model, proposed by Priestly et al. [6], is used in this study. The model is able to account for the shear strength degradation with increasing curvature ductility demand that can be defined as:

$$
V_{u}=k_{\varphi} \sqrt{f_{c}^{\prime}}\left(0.80 A_{g}\right)+\frac{A_{v} f_{y v} D^{\prime} \cot 30^{\circ}}{s}+\frac{d-c}{2 L_{a}} N
$$

Where $k_{\varphi}$ is a parameter associated the curvature ductility demand $\mu_{\varphi}[1], f_{c}^{\prime}$ is concrete strength , $A_{v}$ is the area of the transverse reinforcement, $f_{y v}$ is the yield strength of transverse reinforcement, $D^{\prime}$ is the distance measured parallel to the applied shear between the centres of transverse reinforcement, $s$ is the spacing of transverse reinforcement, $d$ is the section depth and $c$ is the neutral axis depth respectively.

The slope of the second and third branch $\left(G A_{1}\right)$ can be expressed as:

$$
G A_{1}=\frac{V_{u 0}-V_{c r}}{\gamma_{s t}-\gamma_{c r}}
$$

Where $V_{u 0}$ is the non-degraded shear strength that can determine from Eq. (4) by setting the curvature ductility demand which becomes lesser than 3 .

The shear strain at the onset of transverse reinforcement yielding $\left(\gamma_{s t}\right)$ can simply be determined by using the truss analogy. However, it is not corresponding well with experimental results due to the axial load and aspect ratio effects. Mergos and Kappos [1] suggested two modification factors $(\kappa$ and $\lambda)$ to improve the accuracy of these equations. The value of $\gamma_{s t}$ was given by

$$
\gamma_{\text {truss }}=\frac{V_{c r}}{G A_{0}}+\frac{A_{v} f_{v v}\left(\sin ^{4} 45^{\circ}+\frac{E_{s}}{E_{c}} p_{v}\right)}{s E_{s} b p_{v} \sin ^{4} 45^{\circ} \cot 45^{\circ}}
$$

$$
\begin{gathered}
\kappa=1-1.07 \nu \text { and } \lambda=5.37-1.59 \min \left(2.5, L_{a} / h\right) \\
\gamma_{s t}=\kappa \lambda \gamma_{\text {truss }}
\end{gathered}
$$

Where $\gamma_{\text {trus }}$ is the shear associated with the yielding of transverse reinforcement based on truss anology, $E_{s}$ is the elastic modulus of steel, $E_{c}$ is the elastic modulus of concrete, $p_{v}$ is the volumetric ratio of transverse reinforcement, $b$ is the section width and $v=N /\left(f_{c}^{\prime} A_{g}\right)$ is the normalized compressive axial load.

Finally, the last branch cd represents the plastic behavior of shear and connects the shear-yielding point c $\left(V_{u 0}, \gamma_{s t}\right)$ to the ultimate point $\mathrm{d}\left(V_{u 0}, \gamma_{u}\right)$ which associates with the onset of shear failure. The value of $\gamma_{u}$ is based on regression analysis of experimental results and suggested by Mergos and Kappos [1]:

$$
\begin{gathered}
\lambda_{1}=1.0-2.5 \min (0.40, v) \text { and } \lambda_{2}=\min \left(2.5, L_{a} / h\right)^{2.0} \\
\lambda_{3}=0.31+17.8 \min \left(\left(A_{v} f_{y v}\right) /\left(b s f_{c}{ }^{\prime}\right), 0.08\right) \\
\gamma_{u}=\lambda_{1} \lambda_{2} \lambda_{3} \gamma_{s t} \geq \gamma_{s t}
\end{gathered}
$$

Where $\lambda_{1}, \lambda_{2}$ and $\lambda_{3}$ are the parameter acounting for axial load, aspect ratio and amount of transverse reinforcement respectively. It is important to note that the Mergos and Kappos formula for $\gamma_{s t}$ and $\gamma_{u}$ estimation suggested the criteria: $1.11 \leq L_{a} / h \leq 3.91$, $0 \leq v \leq 0.61$ and $0.47 \% \leq\left(A_{v} f_{y v}\right) /\left(b s f_{c}^{\prime}\right) \leq 8.13 \%$ [1].

\subsection{The modified Mergos-Kappos shear-flexure interaction procedure (MMKSFIP)}

According to the primary curve, there are two phenomena results due to the shear-flexure interaction effect on the undamaged primary curve. The first one is the degradation of the shear strength because of the increasing curvature ductility demand as defined by the shear strength model in Eq. (4). From this result, the last branch cd is degraded from the undamaged primary curve to the new portion that associates with the shear strength at the start of load increment step. Moreover, the reduction of the shear strength also leads to the degradation of the shear stiffness as defined in the second phenomena result. The authors refer to the degraded shear stiffness as "the effective shear stiffness $(G A)_{e f f}$ ". As shown in Fig. 1, there are three cases to be confronted when the shear-flexure interaction appears after the flexural reinforcement yielding.

The first case refers to the case of the nondegradation of the shear strength, the sectional curvature ductility is lesser than 3 that based on the shear strength model in Eq. (4). In this case (Fig.1), the sectional shear force at the start and end of load increment step are located on the undamaged primary curve with the cracked sectional shear stiffness $\left(G A_{1}\right)$.

The second case presents the case of the degradation of the shear strength for the first time, the sectional curvature ductility is larger than 3 for the first time. From this case, the sectional shear force at the start of 
load increment step lies on the undamaged primary curve but the sectional shear force at the end of load increment step is situated on the damage primary curve due to the shear-flexure interaction effect with the effective shear stiffness $\left((G A)_{e f f}\right)$ as shown in Fig.1.

The last case shows the case after the degradation of the shear strength for the first time. The sectional shear force at the start and end of load increment step are located on the damaged primary curve with the effective shear stiffness $\left((G A)_{\text {eff }}\right)$.

According to the effective shear stiffness estimation, the original procedure proposed by Mergos and Kappos [1] is adopted and modified herein. The authors refer to this procedure as "modified Mergos-Kappos shearflexure interaction procedure (MMKSFIP)". From all three possible cases, The incremental sectional shear force $(\Delta V)$ and the effective shear stiffness $\left((G A)_{e f f}\right)$ can determine form a given incremental sectional shear strain $(\Delta \gamma)$ by using the MMKSFIP through the proposed reference slope that links the sectional shear force at the start of load increment step to the sectional shear force at the end of load increment step on the undamaged primary curve as shown in Fig. 1. In this procedure, the so-called reference sectional shear stiffness $\left(\left(G A_{r e f}\right)_{i}^{k}\right)$ can define as:

$$
\left(G A_{r e f}\right)_{i}^{k}=\frac{V_{0, i}^{k+1}-V^{k}}{\Delta \gamma_{i}^{k}}
$$

Where $V_{0, i}^{k+1}$ is the non-degradation sectional shear force that correponds to the sectional shear strain $\gamma_{i}^{k+1}=\gamma_{i}^{k}+\Delta \gamma_{i}^{k}$ and can be written as:

$$
V_{0, i}^{k+1}=V_{c r}+G A_{1}\left(\gamma_{i}^{k+1}-\gamma_{c r}\right)
$$

It is worth noting that, the reference sectional shear stiffness $\left(\left(G A_{r e f}\right)_{i}^{k}\right)$ in the first and second cases is simply equal to the cracked sectional shear stiffness $\left(G A_{1}\right)$ as shown in the Fig. 1. Considering the relation between incremental sectional shear force $(\Delta V)$ and shear strain $(\Delta \gamma)$ in Fig. 1 leads to the following expression:

$$
\Delta \gamma_{i}^{k}=\frac{\Delta V_{i}^{k}}{\left(G A_{e f f}\right)_{i}^{k}}=\frac{\Delta V_{i}^{k}+\left(\Delta V_{c}^{\mathrm{deg}}\right)_{i}^{k}}{\left(G A_{r e f}\right)_{i}^{k}}
$$

Where $\left(\Delta V_{c}^{\mathrm{deg}}\right)_{i}^{k}$ is the degradation of the concrete shear resisting mechanism can calculate by:

$$
\left(\Delta V_{c}^{\mathrm{deg}}\right)_{i}^{k}=\left(G A_{r e f}\right)_{i}^{k} \Delta \gamma_{i}^{k}-\frac{\left(V_{u, i}^{k}-V_{i}^{k}\right)}{\left(\gamma_{s t}-\gamma_{i}^{k}\right)} \Delta \gamma_{i}^{k}
$$

The effective shear stiffness $\left(\left(G A_{\text {eff }}\right)_{i}^{k}\right)$ can calculate by solving Eq. (13). The following equation is obtained.

$$
\left(G A_{e f f}\right)_{i}^{k}=\frac{\Delta V_{i}^{k}}{\Delta V_{i}^{k}+\left(\Delta V_{c}^{\mathrm{deg}}\right)_{i}^{k}}\left(G A_{r e f}\right)_{i}^{k}
$$

It is observed from Eq. (15) that the effective shear stiffness $\left(\left(G A_{\text {eff }}\right)_{i}^{k}\right)$ is a function of the incremental sectional shear force $\left(\Delta V_{i}^{k}\right)$ but both quantities are unknown. To overcome this problem, an iterative analytical scheme is applied within the iterative step $i$ of the load increment $k$. It needs to note that the following parameters $\Delta \gamma_{i}^{k},\left(G A_{r e f}\right)_{i}^{k}$ and $\left(\Delta V_{c}^{\mathrm{deg}}\right)_{i}^{k}$ aren't changed during an iterative scheme except $\left(G A_{e f f}\right)_{i}^{k}$ and $\Delta V_{i}^{k}$. For this reason, the additional subscript " $j$ " is added into $\left(G A_{\text {eff }}\right)_{i}^{k}$ and $\Delta V_{i}^{k}$ to define the iterative step of the shearflexural interaction process. The iterative process within the shear-flexural interaction procedure in this study is adopted from Newton-Raphson iterative technique. Firstly, an initial effective shear stiffness $\left(\left(G A_{e f f}\right)_{i, j=1}^{k}\right)$ is assumed. Shear force increment $\left(\Delta V_{i, j}^{k}\right)$ is calculated from $\Delta V_{i, j}^{k}=\left(G A_{e f f}\right)_{i, j}^{k} \Delta \gamma_{i}^{k}$ in the second step and Eq.(15) is enforced to a residual function by moving $\left(G A_{e f f}\right)_{i, j}^{k}$ into the opposite side in the third step. The fourth step is the derivative of residual function determination. Next, the new value of $\left(G A_{e f f}\right)_{i, j+1}^{k}$ is updated from the residual function and its derivative. Finally, the iterative procedure terminates, when the value of residual function converges with the specified tolerance.

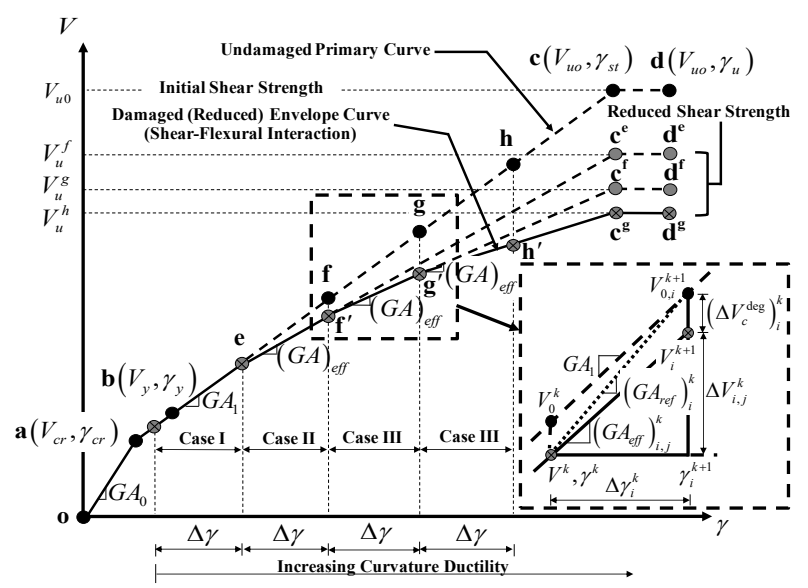

Fig. 1. Primary curve of the shear model

\subsection{The hysteretic shear behavior}

In this study, the hysteretic relation between the shear force and shear strain is based on the uniaxial behaviors and expressed in term of shear strain. Moreover, the hysteretic law is adapted from the hysteretic momentrotation relation with pinching and damage, proposed by Filippou et. al. [7]. It comprises of three parts. The first one is the monotonic envelop curve (Primary curve). The second and third parts are unloading and reloading curves respectively.

\section{Numerical example}

To verify the accuracy and efficiency of the proposed model, a numerical example is presented herein. The example is presented by using 16 elements with 7 GaussLobatto integration points under the displacement-based 
method that are enough for the convergent responses. Moreover, the frame section is discretized into 40 fibers (Layers) to represent the nonlinear responses under the fiber-section model.

The specimen 3SMD12 was one of the full-scale R/C column tests by Lynn [8]. The column was a double curvature specimen under a constant axial load and cyclic lateral displacements at the end of the column and collapsed in shear after the longitudinal reinforcement bars yielding. It's so-called "Flexure-shear failure". The initial shear strength can predict from Eq. (4) about $597.93 \mathrm{kN}$ by using the properties in Table 1.

Table 1. Properties of specimen 3SMD12

\begin{tabular}{|c|c|c|c|}
\hline Properties & Value & Properties & Value \\
\hline$f_{c}^{\prime}$ & $25.5(\mathrm{MPa})$ & $s$ & $305(\mathrm{~mm})$ \\
\hline$b$ and $h$ & $457.2(\mathrm{~mm})$ & $p_{v}$ & 0.0017 \\
\hline$f_{y l}$ & $331(\mathrm{MPa})$ & $L$ & $2,946(\mathrm{~mm})$ \\
\hline$f_{y v}$ & $400(\mathrm{MPa})$ & $N$ & $1,512(\mathrm{kN})$ \\
\hline
\end{tabular}

Fig. 2 shows the lateral load vs. lateral displacement response as derived by the proposed model and the experimental data. It can be seen that the proposed model is able to represent the complex behavior of the experimental results very well. These include the member capacity, stiffness degradation with increasing displacement amplitude, amount of dissipated hysteretic energy and general shape of hysteretic response. Moreover, the proposed model can capture the degradation of the shear strength and shear stiffness due to the increasing curvature ductility demand before the shear failure occurs at the displacement about $17.12 \mathrm{~mm}$.

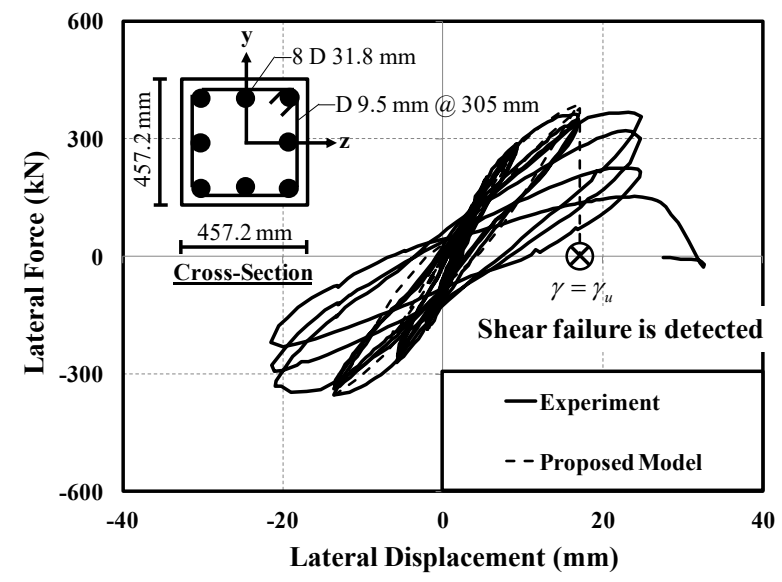

Fig. 2. The lateral load and total displacement relationship between the proposed model and experimental data

Fig. 3. presents the shear force vs. shear strain hysteretic relationship of a specimen 3SMD12 at the end of a column (Inside plastic hinge regions). It can be observed that after the curvature ductility demand value exceeds 3 , the shear strain increases immediately and shear stiffness is degraded from the undamaged primary curve into the damaged primary curve. Finally, the models can predict shear failure when the shear strain reaches $\gamma_{u}$, that is found to be $3.33 \times 10^{-3}$.

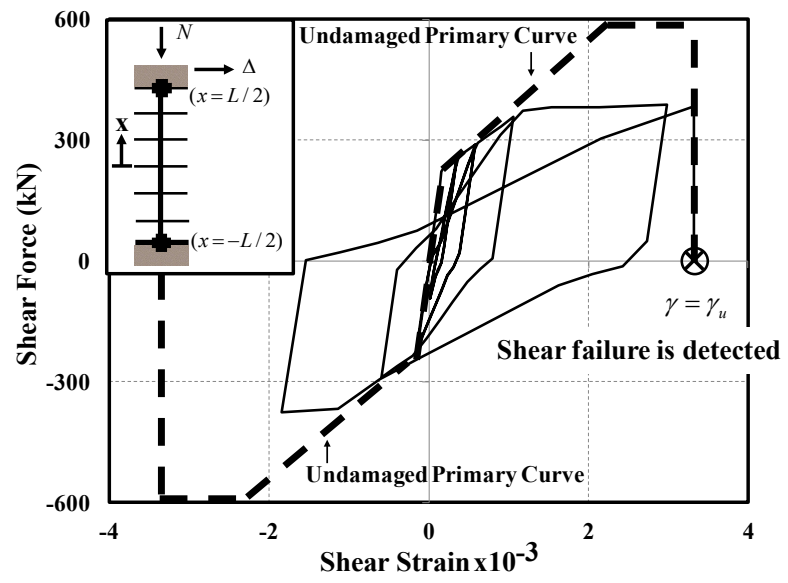

Fig. 3. Shear force vs. shear strain hysteresis loops at the end of a column (inside plastic hinges)

\section{Conclusions}

This paper presents an alternative of the shear model for the $\mathrm{R} / \mathrm{C}$ frame structures analysis under monotonic and cyclic loading. The degradation of the shear strength and shear stiffness due to the shear-flexure interaction effect is included in this model. Moreover, the modified Mergos-Kappos shear-flexure interaction procedure (MMKSFIP) is proposed here and used to estimate the effective shear stiffness. Finally, the response analysis of a numerical example confirms the accuracy and computational efficiency of the proposed model.

\section{Funding}

This study was partially supported by Faculty of engineering, Prince of Sonkla University.

\section{References}

1. P.E. Mergos, A.J. Kappos, Eng. Struct., 44, 94-106 (2012)

2. P. Ceresa, L. Petrini, R. Pinho, IUSS, Pavia, Italy (2008)

3. F.J. Vecchio, M.P. Collins, ACI Struct. J., 83, 219231 (1986)

4. P.E. Mergos, A.J. Kappos, Earthquake Eng. Struct. Dyn., 37, 1349-1370 (2008)

5. R.L. Taylor, User manual: version 7.3 (Department of Civil and Environmental Engineering, UC, Berkeley, 2000)

6. M.J.N. Priestley, F. Seible, R. Verma,Y. Xiao, Report no SSRP-93/06 (USD, Calif., 1993)

7. F.C. Filippou, E.P. Popov, V.V. Bertero, EERC Report 83-19 (EERC, UC, Berkeley, 1983)

8. A. Lynn, Ph.D. thesis (UCB, Berkeley, Calif., 2001) 